\title{
A recognition algorithm of VGPO jamming based on discrete chirp-Fourier transform
}

\author{
Chuanzhang Wu and Baixiao Chen*
}

${ }^{*}$ Correspondence:

bxchen@xidian.edu.cn

National Laboratory of Radar Signal

Processing, Xidian University, Taibai

South Road, Xi'an, 710071 China

\begin{abstract}
This paper addresses the recognition problem of velocity gate pull-off (VGPO) jamming from the target echo signal for the velocity-based tracking system. The discrete chirp-Fouriertransform (DCFT) is studied in this paper to jointly estimate the chirp rates and frequencies of the target and jamming signals. Firstly, the scaling characteristic of the DCFT algorithm is explored. Then, we focus on the quantitative effect of the VGPO jamming signal by analyzing the jointly estimated results in each pulse. The quantitative effect indicates that, as long as the estimated frequency is unchanged, the relationship between the estimated chirp rate and the pulse numbers is similar to the relationship between the frequency offset of VGPO jamming and the time. Finally, by utilizing the joint estimated results in each pulse repetition interval and calculating the mean square to variance ratio (MSVR) of the normalized estimated chirp rate, the VGPO jamming can be recognized. Simulation results show that the jamming signal and the target echo become distinguishable with the proposed feature. Comparing to the existing works, the proposed method can correctly recognize the jamming signal with lower jamming-to-noise ratio (JNR) $5 \mathrm{~dB}$ with less data needed, which means it can work effectively in the early stage of interference implementation and shows great potential in practical applications.
\end{abstract}

Keywords: Deceptive jamming, Discrete chirp-Fourier transform, Jamming recognition, Feature extraction

\section{Introduction}

Electronic countermeasure (ECM) has always been an important research direction in radar $[1,2]$. As a typical category, the deceptive jamming is often used to deceive a hostile radar system by generating a large number of active false targets in terms of direction, position, and velocity [3]. With the widespread application of the digital radio frequency memory (DRFM) technique, the interference effect of ECM is further reinforced. In a DRFM system, the intercepted radar signal is firstly down-shifted in frequency. And then it is sampled with an analog-to-digital converter and stored in a high-speed digital memory where the samples can be manipulated in amplitude, frequency, and phase to produce

(c) The Author(s). 2020 Open Access This article is licensed under a Creative Commons Attribution 4.0 International License, which permits use, sharing, adaptation, distribution and reproduction in any medium or format, as long as you give appropriate credit to the original author(s) and the source, provide a link to the Creative Commons licence, and indicate if changes were made. The images or other third party material in this article are included in the article's Creative Commons licence, unless indicated otherwise in a credit line to the material. If material is not included in the article's Creative Commons licence and your intended use is not permitted by statutory regulation or exceeds the permitted use, you will need to obtain permission directly from the copyright holder. To view a copy of this licence, visit http://creativecommons.org/licenses/by/4.0/. 
desired deceptive signals. When being recalled, the stored samples are processed by a digital-to-analog converter and then transmitted back to the victim radar [4].

Over several decades, with the continuous development of ECM, the electronic counter-countermeasures (ECCM) are also studied by more and more researchers. Many methods for recognizing and countering deceptive jamming are studied based on the DRFM technique. In 2006, Maria analyzed the phase and delay quantization effect of the DRFM device in detail [5]. In [6], she further defined the difference between the deceptive jamming and the target echo in the frequency domain as the jamming signal error angle which is introduced by the DRFM system and proposed a detection method.

Furthermore, many researchers counter the jamming signal from the aspect of jamming detection. In [7], the authors presented the adaptive detection schemes and the enhanced adaptive sidelobe blanking algorithms, which consider the realistic scenario when a mismatched signal is present in the data. In [8], they proposed a new algorithm to detect the coherent signals from noise, clutter, and noise-like interference. For the same problem, two datasets are utilized at the design stage to get receiver adaption, and a two-stage detector is recommended [9]. In [10], the authors conceived a multiple-stage adaptive architecture to detect the target, and show a better performance compared to the two-stage detector in [9].

What is more, the jamming signal can also be distinguished from the target according to their difference in various domains. In [11], the authors found the difference between the deceptive jamming and the target echo by calculating their product spectrum matrix. In [12], the deceptive jamming is analyzed in the spatial frequency domain in the multiple-input multiple-output system with frequency diverse array and suppressed in the joint transmit-receive domain. Moreover, the deceptive jamming and its anti-jamming methods in some new radar systems are also studied [13, 14].

Although the range gate pull-off (RGPO) in DRFM devices has been studied by many researchers $[5,15]$, not many published literature on the velocity gate pulloff (VGPO) jamming about the recognition and countering problem are available. In [16], the authors proposed an approach to suppress the velocity deception jamming by designing adaptive initial phases in the pulse repetition interval (PRI) domain. This method is further applied in the cognitive radar system [17]. In [18], a VGPO jamming countermeasure is proposed by utilizing the front-back-edge tracking gate, while it has to judge the jamming pulling off direction first. These methods are based on waveform design and radar systems, respectively. By drawing on the texture feature in image recognition, Yang converted the time-frequency image of the jamming signal into a gray image and then extracted the image feature based on the Zernike moments and recognize the jamming signal [19]. Although this method can recognize various jamming signals in a single pulse, it requires that there are some distinct differences in the time-frequency domain among various kinds of jamming signals. In other words, it can only be effective after the pull-off jamming has been implemented for a long time.

The purpose of this paper is to approach a recognition algorithm towards the VGPO jamming. To this end, the joint estimation algorithm of the chirp rate and frequency is introduced. Different from the method in [19], we analyze the theoretical estimation results of chirp rate and frequency in each pulse and then extract the feature about the estimated chirp rate to distinguish the VGPO jamming and target. 
Currently, many methods are available on joint estimation, such as maximumlikelihood [20], adaptive chirplet transform [21], and phase function [22]. However, the theoretical value of the rate of frequency of the jamming signal is far less than the minimum interval in the chirp rate domain in a general way. Consequently, most of these methods will lose efficacy. For the discrete chirp-Fourier transform (DCFT) algorithm[23], it is generalized from the discrete Fourier transform (DFT). Besides matching the multiple frequencies similar to the DFT algorithm, it uses the multiple chirp components to match the chirp rates. Most important of all, it can increase the resolution in the chirp rate domain by introducing a scaling factor, which is termed as the scaling property and will be analyzed in this paper. As a result, it is still applicable in this situation. Nowadays, it has applied in many cases [24, 25].

This paper is organized as follows. In Section 2, the received signal models of both real target echo and VGPO jamming are first formulated; then, we introduce the modified DCFT and analyze its scaling property, derive the estimated chirp rate and frequency in each pulse repetition interval (PRI), and further define a new feature parameter. Section 3 shows the simulation results. Section 4 is the concluding remarks.

\section{Methods}

In this section, we elaborate on the theory and the method of jamming signal recognition. First, we present the signal models for VGPO jamming and target echo. Second, we introduce the modified DCFT and study its scaling property. Then, the quantitative effect is analyzed when performing the joint estimation with the DCFT algorithm, and it is also discussed in the non-linear scenario. Finally, we define a feature to distinguish the VGPO jamming and the target echo.

\subsection{Signal model}

The whole process that a jammer implements VGPO interference can be divided into three steps: pull-off halting, pulling off, and closing [3]. After the radar signal is intercepted, the jammer retransmits the signal with a Doppler frequency shift inflicted on. To deceive the radar effectively, the power of the jamming signal is always greater than that of the real target echo. Therefore, the tracking loop of the radar would track the false Doppler frequency and be slowly pulled away from the velocity gate of the real target.

Assume the signal transmitted by radar is $s(t)$. Then, the signal reflected by a hypothetical target in the far-field can be modeled as [16]

$$
s_{r}(t)=A_{r} s\left(t-t_{r}\right) e^{j 2 \pi f_{d}\left(t-t_{r}\right)}+n(t)
$$

where $A_{r}$ and $t_{r}$ denote the reflection coefficient and the time delay of the hypothetical target, respectively, and $f_{d}$ is its Doppler frequency.

Comparing with the real target echo, the VGPO jamming has a pseudo-Doppler frequency shift based on $f_{d}$. For convenience, we consider the scenario that only one interference exits, and the jamming signal can be expressed as

$$
s_{J}(t)=A_{J} s\left(t-t_{J}\right) e^{j 2 \pi f_{d j}\left(t-t_{J}\right)}+n(t)
$$

where $A_{J}$ denotes the equivalent reflection coefficient of the interfering source, $t_{J}=t_{r}+$ $\Delta t_{d}$ is the jamming propagation delay, and $\Delta t_{d}$ is the time delay of jammer relay which is often far less than $t_{r}, f_{d j}=f_{d}+\Delta f_{d}$ is the modulated pseudo-Doppler frequency. Here, we 
define jamming-to-noise ratio (JNR) $A_{J}^{2} / \sigma^{2}$, and signal-to-noise ratio (SNR) $A_{r}^{2} / \sigma^{2}$, where $\sigma^{2}$ is the variance of Gaussian noise $n(t)$, and jamming-to-signal ratio (JSR) is defined as JNR-SNR.

Similar to the RGPO system described in [5, 26], a VGPO system should linearly increase the frequency offset to create a series of constant velocity-rate false targets. This scenario is also the one we often use when analyzing VGPO $[19,27,28]$ and is equivalent to the constant acceleration profile in the range domain. Then, the $f_{d j}$ at the $m$ th PRI can be expressed as [28]:

$$
f_{d j}=V_{f}\left[t+(m-1) T_{r}\right]+f_{d}
$$

where $V_{f}$ denotes the slope of $f_{d}$ with respect to $t$ which is also called acceleration and $T_{r}$ denotes the pulse repetition period.

To enable radar to accurately capture and track the velocity loop of the jamming signal, $V_{f}$ would not be greater than the maximum trackable acceleration of a target to radar [1]. On the other side, $V_{f}$ would not be particularly small for implementing interference effectively and protecting the real target. In a word, $V_{f}$ has a reasonable value range.

Substitute (3) into (2), and we can find that, despite the signal form, the VGPO jamming signal is a chirp function with a chirp rate of $V_{f}$. This is the inspiration for the jamming identification in this paper.

As the specific form of $s(t)$ is not assumed, we may have to do some preprocessing to prevent the influence of the potential time-varying frequency components in signal form on chirp rate estimation. The preprocessing may be different for various kinds of signals. Take the widely used linear frequency modulation (LFM) signal as an example, it has a quadratic term in terms of $t$, i.e., the inherent chirp rate. Generally speaking, the acceleration $V_{f}$ of the VGPO jamming (three times the acceleration of gravity in Xband is about $2 \mathrm{kHz} / \mathrm{s}$ ) is much smaller than the chirp rate of LFM signal (typical value $\left.10^{10}-10^{14} \mathrm{~Hz} / \mathrm{s},[1]\right)$. As a result, the de-chirping processing should be performed first in the recognition procedure for the radar that emits LFM signal.

After being sampled with discrete-time $t=n T_{s}$ where $T_{s}$ denotes the sampling interval, and performing some appropriate preprocessing, the jamming signal $s_{J}^{\prime}(t)$ in $m$ th PRI can be expressed as

$$
\begin{aligned}
s_{J}(n) & =\left.s_{J}^{\prime}(t)\right|_{t=n T_{s}} \\
& =A_{J} \exp \left\{j 2 \pi\left(V_{f} n^{2} T_{s}^{2}+f_{d j}^{\prime} n T_{s}\right)+j \varphi_{j}\right\},
\end{aligned}
$$

where

$$
\begin{gathered}
f_{d j}^{\prime}=f_{d}+(m-1) V_{f} T_{r}-V_{f} t_{J}+f_{d 0}, \\
\varphi_{j}=-2 \pi\left(f_{d}+(m-1) V_{f} T_{r}\right) t_{J}+\varphi_{0},
\end{gathered}
$$

where $f_{d 0}$ and $\varphi_{0}$ separately denote the time-invariant frequency and constant phase term in $s_{J}(n)$.

The phase of the discretized jamming signal in (4) contains three components: a quadratic item with respect to $n T_{s}$ with a coefficient $V_{f}$, a linear item with respect to $n T_{s}$ with a coefficient $f_{d j}^{\prime}$, and a time-independent item $\varphi_{j}$. Ignoring the time-independent phase item, the coefficient $f_{d j}^{\prime}$ of the linear item is different in various PRIs, while the coefficient of the quadratic phase items is the same. However, the following analysis indicates 
that the quantization in the frequency domain will influence the estimated result in the chirp rate domain when performing joint estimation to the jamming signal.

\subsection{Discrete chirp-Fourier transform and its scaling property}

The discrete chirp-Fourier transform algorithm is used to jointly estimate the chirp rate and the frequency of a chirp-type signal. For an analog chirp signal $x(t)=\exp \left[j 2 \pi\left(k_{0} t+\right.\right.$ $\left.\left.l_{0} t^{2}\right)\right]\left(0 \leq k_{0}, l_{0} \leq N-1\right)$, its discretized form with sample rate $1 / N$ is

$$
x_{I}(n)=W_{N}^{-\left(k_{0} n+l_{0} n^{2} / N\right)}
$$

where $W_{N}=\exp (-j 2 \pi / N)$. The corresponding $N$-point DCFT of signal $x_{I}(n)$ is defined as [29]

$$
X_{c}(k, l)=\frac{1}{\sqrt{N}} \sum_{n=0}^{N-1} x_{I}(n) W_{N}^{k n+l n^{2} / N}, 0 \leq k, l \leq N-1
$$

where $k$ and $l$ represent the frequency and chirp rate variables, respectively.

In (7), the duration of the signal is normalized as 1, which makes both of $k$ and $l$ less equal than the length of signal $N$. In radar signal processing, the Doppler frequency of the signal is less than the sampling frequency of $f_{s}$, and the chirp rate is less than $f_{s}^{2}$ correspondingly. After sampling with discrete-time $t=n T_{s}$, the discretized form of $x(t)$ is rewritten as

$$
x(n)=\exp \left[j 2 \pi\left(k_{0} n T_{s}+l_{0} n^{2} T_{s}^{2}\right)\right]
$$

where $0 \leq k_{0}<f_{s}, 0 \leq l_{0}<f_{s}^{2}$. To maintain the consistency between the estimated values of frequency and chirp rate and the actual ones, the corresponding form of DCFT should be modified as

$$
X_{m c}(k, l)=\frac{1}{\sqrt{N}} \sum_{n=0}^{N-1} x(n) W_{N}^{k n+l n^{2}}, 0 \leq k, l \leq N-1
$$

Each bin in frequency domain and chirp rate domain represents $f_{s} / N$ and $f_{s}^{2} / N$, respectively. When $\left|X_{m c}(k, l)\right|$ maximizes, the estimated values of $k$ and $l$ are

$$
k=k_{0} N T_{s}, l=l_{0} N T_{s}^{2}
$$

respectively, and the corresponding estimated values of frequency and chirp rate are $k_{0}$ and $l_{0}$, respectively.

Essentially, the time delay of a signal is equivalent to zero padding in front of the signal. It changes the phase of the signal's DFT result, while the estimated frequency and the envelope of DFT results are unchanged. In a very short timestamp, the positions of target and jammer are usually considered to be located in the same range cell. Under this assumption, both of the time delay $t_{r}$ and $t_{J}$ are constant, which means the number of zero paddings in each PRI is the same during this period. In other words, the time delay in these PRIs would not influence the estimated value of frequency through DFT. As the DCFT algorithm is a generalized form of the DFT algorithm, this conclusion is applicable for jointly estimating the values of chirp rate and frequency with the DCFT algorithm.

From (10), one can see that the chirp rate variable only appears in the factor $W_{N}^{l h^{2}}$. It means that the chirp rate domain of DCFT can be broadened or compressed after dividing the variable $l$ by a scaling factor $C$, which equivalently increases or decreases the minimum interval in the chirp rate axis. According to the scalability of the DCFT algorithm 
in the chirp rate domain, we further define the modified DCFT as follows:

$$
X_{m c}^{\prime}(k, l)=\frac{1}{\sqrt{N}} \sum_{n=0}^{N-1} x(n) W_{N}^{k n+l n^{2} / C}
$$

At this time, the maximum value in the chirp rate axis changes from $f_{s}^{2}$ to $f_{s}^{2} / C$, and the corresponding minimum interval decreases to $f_{s}^{2} / N C$. Similarly, for the chirp signal in (9), the peak position in the modified DCFT domain is located at

$$
k=k_{0} N T_{s}, l=l_{0} N C T_{s}^{2},
$$

and the corresponding values of frequency and chirp rate are $k_{0}$ and $l_{0}$, respectively. It indicates that the estimated values of chirp rate and frequency are unchanged in the modified DCFT compared with the DCFT in (10).

As mentioned above, the value of $V_{f}$ is in a reasonable range. What is more, the sampling frequency $f_{s}$ is always known as prior information. As a result, the scaling factor $C$ has a rough estimation for the radar side. The bigger the scaling factor $C$, the smaller the minimum interval in the chirp rate domain for the modified DCFT algorithm. In fact, if $C$ is big enough or much bigger than $N$, the DCFT result for different $l$ is almost the same. In this case, the DCFT algorithm is considered as degenerating into the DFT algorithm and could cause a huge estimation error to the chirp rate at low SNR.

\subsection{The analysis of quantitative effect}

In this part, we analyze the influence of a small frequency offset on the DCFT estimation results. In the DFT algorithm, we estimate the constant frequency $f_{n 0}$ of a signal by matching it with a quantitative value in frequency domain, i.e., $f_{n 0}=n_{0} f_{s} / N$, where $N$ is the total number of DFT and $n_{0}\left(0 \leq n_{0}<N-1\right)$ is a certain nonnegative integer. When the signal's frequency $f_{n 1}$ has an offset $\Delta f$ based on $f_{n 0}$, i.e.,

$$
f_{n 1}=f_{n 0}+\Delta f
$$

the peak still exhibits at the position $f=f_{n 0}$ only if $|\Delta f|<f_{s} / 2 N$. It means the frequency offset $\Delta f$ is ignored due to the quantization in the frequency domain.

Similarly, the quantization error must occur in the DCFT algorithm as well. From [23], it is pointed out that, for a discrete chirp signal

$$
\tilde{x}(n)=W_{N}^{-\left(\tilde{k}_{0} n+\tilde{l}_{0} n^{2}\right)},
$$

its DCFT result has the similar peak property as the signal $x(n)=W_{N}^{-\left(k_{0} n+l_{0} n^{2}\right)}$, where $\tilde{k}_{0}$ and $\tilde{l}_{0}$ are close to integers $l_{0}$ and $k_{0}$, respectively, i.e.

$$
\left|\tilde{l}_{0}-l_{0}\right|<\epsilon \text { and }\left|\tilde{k}_{0}-k_{0}\right|<\eta
$$

where $\epsilon$ and $\eta$ are two arbitrary positive values. However, this conclusion is drawn based on the condition that $\tilde{l}_{0}$ and $\tilde{k}_{0}$ are close enough to $l_{0}$ and $k_{0}$, i.e., $\epsilon, \eta \approx 0$. As the process of pulling off a velocity gate is continuous, the pseudo-Doppler frequency of the jamming signal changes in each PRI. Moreover, the minimum interval in the chirp rate axis decreases after adopting the modified DCFT in (12). As a result, it becomes very necessary to analyze the quantitative effect of the DCFT algorithm in this paper. 
For a discrete VGPO jamming in (4), its modified DCFT after ignoring the timeindependent phase item $\varphi_{j}$ can be calculated by

$$
\begin{aligned}
X_{m c}^{\prime}(k, l) & =\frac{1}{\sqrt{N}} \sum_{n=0}^{N-1} s_{J}(n) W_{N}^{k n+l n^{2} / C} \\
& =\frac{A_{J}}{\sqrt{N}} \sum_{n=0}^{N-1} e^{j 2 \pi\left(V_{f} n^{2} T_{s}^{2}+f_{d j}^{\prime} n T_{s}-k n / N-l n^{2} / N C\right)}
\end{aligned}
$$

Let the Doppler frequency

$$
f_{d j}^{\prime}=\tilde{f}_{d j}+\Delta f_{m}
$$

where $\tilde{f}_{d j}$ is an integer multiple of $f_{s} / N C$, and $\Delta f_{m}$ denotes the remainder Doppler frequency besides $\tilde{f}_{d j}$ in $m$ th PRI. Here, we assume the total number of pulses is $M$ when the estimated frequency through the modified DCFT is fixed as $\tilde{f}_{d j}$. Then, we have $0 \leq m \leq M-1$ and $0 \leq \Delta f_{m}<\Delta f_{M}<f_{s} / N C$.

For the convenience of representation, we let $\tilde{f}_{d j}=f_{d}-V_{f}\left(T_{r}+t_{J}\right)+f_{d 0}$, and $\Delta f_{m}=$ $m V_{f} T_{r}$ according to (5) and (18). Then, (17) can be rewritten as follows:

$$
X_{m c}^{\prime}(k, l)=\frac{A_{J}}{\sqrt{N}} \sum_{n=0}^{N-1} e^{j 2 \pi \phi_{1}} e^{j 2 \pi \phi_{2}},
$$

where $\phi_{1}(n)=\left(V_{f} T_{s}^{2}-\frac{l}{N C}\right) n^{2}+m n V_{f} T_{r} T_{s}$ and $\phi_{2}(n)=\left(\tilde{f}_{d j} T_{s}-k / N\right) n$.

Let

$$
P=\left|\sum_{n=0}^{N-1} e^{j 2 \pi \phi_{1}} e^{j 2 \pi \phi_{2}}\right| .
$$

In the complex coordinate system, each complex exponential can be regarded as a unit vector. Consequently, $P$ can be considered as the modulus of a vector summed by $N$ unit vectors, and it reaches to maximum of $N$ when all of the vectors point in the same direction. According to [23], $P$ maximizes when the frequency variation of DCFT matches to the quantized frequency $\tilde{f}_{d j}$, i.e., $\phi_{2}=0$. Then, we have

$$
k=N \tilde{f}_{d j} / f_{s}
$$

On the other side, $\phi_{1}(n)$ differs by an integer to various $n$,

$$
\phi_{1}\left(n_{1}\right)=\phi_{1}\left(n_{2}\right)+\varphi_{0 n}
$$

where $n_{1}$ and $n_{2}$ are two arbitrary different integers between 0 and $N-1$ and $\varphi_{0 n}$ is an integer. Substituting the expression of $\phi_{1}$ into (22), we can yield

$$
\left(V_{f} T_{s}^{2}-\frac{l}{N C}\right)\left(n_{1}+n_{2}\right)+m V_{f} T_{r} T_{s}=\frac{\varphi_{0 n}}{n_{1}-n_{2}}
$$

Let $\alpha_{1}=\varphi_{0 n} /\left(n_{1}-n_{2}\right)$, and $\alpha_{2}=n_{1}+n_{2}$. Then, if $P$ reaches to maximum $N$, it implies that the variable $l$ to arbitrary $n_{1}$ and $n_{2}$ in (23) is solvable, i.e.,

$$
l=N C\left(V_{f} T_{s}^{2}+\frac{m V_{f} T_{r} T_{s}-\alpha_{1}}{\alpha_{2}}\right)
$$

The effective value of $\phi_{1}$ only exists in the fractional part from 0 to 1 due to the periodicity of the exponential function. Correspondingly, the true value of $l$ is the remainder after dividing by $N$ in (24). In other words, for a fixed $m$, the true value of $l$ should be fixed, too. 
From (24), one can see that $l$ and $m$ are linearly related. In general, not all vectors in (20) point in the same direction, and $P$ cannot reach to maximum N. $\varphi_{0 n}$ in (22) is not an integer anymore to a fixed pair of integers $n_{1}$ and $n_{2}$, which changes the estimated chirp rate of jamming signal in $m$ th PRI. Among various PRIs, $m$ is the unique determining factor that influences the value $P$, and the estimated chirp rate $l$. From this point, $\varphi_{0 n}$ is not an integer either for various pairs of $n_{1}$ and $n_{2}$. However, it can be found from the formulation of $\phi_{1}$ that $\varphi_{0 n}$ is linearly related to $m$ for fixed $n_{1}$ and $n_{2}$. It means $l$ is still proportional to $m$. As

$$
m n V_{f} T_{r} T_{s}=n T_{s} \Delta f_{m}<\frac{n}{N C} \leq \frac{n^{2}}{N C}
$$

the influence of $m$ to $\phi_{1}(n)$ and $l$ is small. Once $V_{f}$ is fixed, all integer pairs $n_{1}$ and $n_{2}$ that make $P$ reach to the maximum value (not $N$ ) are basically determined. Therefore, the slope between $l$ and $m$ can be considered to be unchanged at this time.

More generally, $\Delta f$ includes an extra frequency independent of $m$ (part of $\tilde{f}_{d j}$ ) which results in $\varphi_{0 n}$ is not an integer either. Then, the estimated chirp rate of VGPO jamming in $m$ th PRI may differ from the one in (24), while the linear relationship between $l$ and $m$ still exists.

It can be seen that, when a joint estimation of the VGPO jamming with the DCFT algorithm is performed, the quantization in frequency domain will affect the estimated value of the chirp rate, and the estimated chirp rate is linearly related to the residue of the quantized frequency item.

Unlike in (3), we next discuss a more general scenario that the false target is maneuvering, which means $f_{d j}$ has a non-linear correlation with time $t$. Taking the quadratic relation as an example, $f_{d j}$ changes as follows over time $t$ :

$$
f_{d q j}=A_{f}\left[t+(m-1) T_{r}\right]^{2}+V_{f}\left[t+(m-1) T_{r}\right]+f_{d},
$$

where $A_{f}$ is the cubic coefficient of time $t$.

Substituting the $f_{d j}$ in (2) with $f_{d q j}$, and following the same derivation from (4) 6 and (17) (19), we can calculate the modified DCFT of the jamming signal in non-linear scenario. Similar to (5), the frequency item of the preprocessed jamming signal $f_{d q j}^{\prime}$ can be expressed as follows:

$$
\begin{aligned}
f_{d q j}^{\prime} & =A_{f} n^{2} T_{s}^{2}+\left[V_{f}+2 A_{f}(m-1) T r-A_{f} t_{J}\right] n T_{s} \\
& +\Delta f_{m}^{\prime}+f_{d}-V_{f} t_{J}
\end{aligned}
$$

where

$$
\Delta f_{m}^{\prime}=A_{f}(m-1)^{2} T_{r}^{2}+\left(V_{f}-2 A_{f} t_{J}\right)(m-1) T_{r}
$$

Still, we let $\phi_{1}^{\prime}(n)$ be the function that includes high order terms of $n$ and $\Delta f_{m}^{\prime}$, i.e.,

$$
\begin{aligned}
\phi_{1}^{\prime}(n)= & A_{f} n^{3} T_{s}^{3}+\left[V_{f}+2(m-1) A_{f} T_{r}\right] n^{2} T_{s}^{2} \\
& +\Delta f_{m}^{\prime} n T_{s}-\ln ^{2} / N C .
\end{aligned}
$$

Obviously, besides a cubic item of $n T_{s}$, there is a quadratic item of $n$ with respect to $m$, and a linear item of $n$ with respect to $m^{2}$ in $\phi_{1}^{\prime}(n)$. With the same condition as in (22), we can find that there is a quadratic relation between $l$ and $m$ in this situation. More generally, the relationship between $l$ and $m$ is the same with that between $f_{d j}$ and $t$. Here, we term this phenomenon as a quantitative effect. 
Therefore, it can be concluded that a series of small offsets in frequency would increase the values of the chirp rate in joint estimation. The quantitative effect can be interpreted as, within the timestamp when the estimated quantized frequency is unchanged, the estimated chirp rate of the VGPO jamming through the modified DCFT algorithm increases with a tendency that is the same as the relationship between $f_{d j}$ and $t$.

\subsection{Feature extraction}

In this part, we use an identification parameter to benchmark the quantitative effect. As the pull-off stage of VGPO jamming continues for several seconds [1], the quantitative effect will appear periodically. For the real target echo, the estimated chirp rate through the modified DCFT algorithm is essentially the estimation error caused by thermal noise. Its value equals 0 in an ideal case and randomly changes under noisy environment. By utilizing the distinctive quantitative effect, the VGPO jamming can be distinguished from the real target echo.

Here, we define the feature, the mean square to variance ratio (MSVR) of the normalized estimated chirp rate, and utilize it to distinguish the VGPO jamming and the real target echo. Let $C_{e}(m)$ represent the estimated chirp rate in $m$ th PRI, and its normalized form is yield by

$$
C_{e}^{\prime}(m)=\frac{C_{e}(m)-C_{e}^{\min }}{C_{e}^{\max }-C_{e}^{\min }}
$$

where $C_{e}^{\max }$ and $C_{e}^{\min }$ represent the maximum and minimum of $C_{e}$, respectively.

Let $\mathbf{C}_{e}^{\prime}=\left[C_{e}^{\prime}(1) C_{e}^{\prime}(2) \cdots C_{e}^{\prime}(m)\right]$, and the MSVR is defined as

$$
\operatorname{MSVR}=\frac{\mu_{c}^{2}}{\sigma_{c}^{2}}
$$

where $\mu_{c}$ and $\sigma_{c}$ stand for the mean and the variance of $\mathbf{C}_{e}^{\prime}$, respectively.

From the aforementioned content, it is concluded that $C_{e}(m)$ of VGPO jamming linearly (or non-linearly) increases in $M$ PRIs. Although the estimated chirp rate is normalized from 0 to 1 , its variance becomes smaller and smaller as the JNR increases. On the contrary, all of the elements in $\mathbf{C}_{e}$ of real target echo can be regarded as random, which means its MSVR is steady. Consequently, the VGPO jamming signal and the real target echo can be recognized by setting a proper threshold.

The flowchart of the identification procedure to VGPO jamming based on the DCFT algorithm is provided in Fig. 1. After being sampled, the received signal is preprocessed. Then, the chirp rate of the signal is estimated by the modified DCFT in each pulse. Finally, by calculating the value of MSVR with the estimated results in M PRIs, the VGPO jamming can be identified from the target signal.

\section{Results and discussion}

In this section, we conduct some simulation experiments to illustrate the performance of the proposed method in this article. We assume the waveform of the transmitted signal is LFM. It is worth noting that the pulse numbers are set relatively large in some simulations which are similar to [18]. This is because our method is performed from the time when the interference begins to implement, and the shifted frequency is small at the early pull-off stage and even within a single frequency interval. 


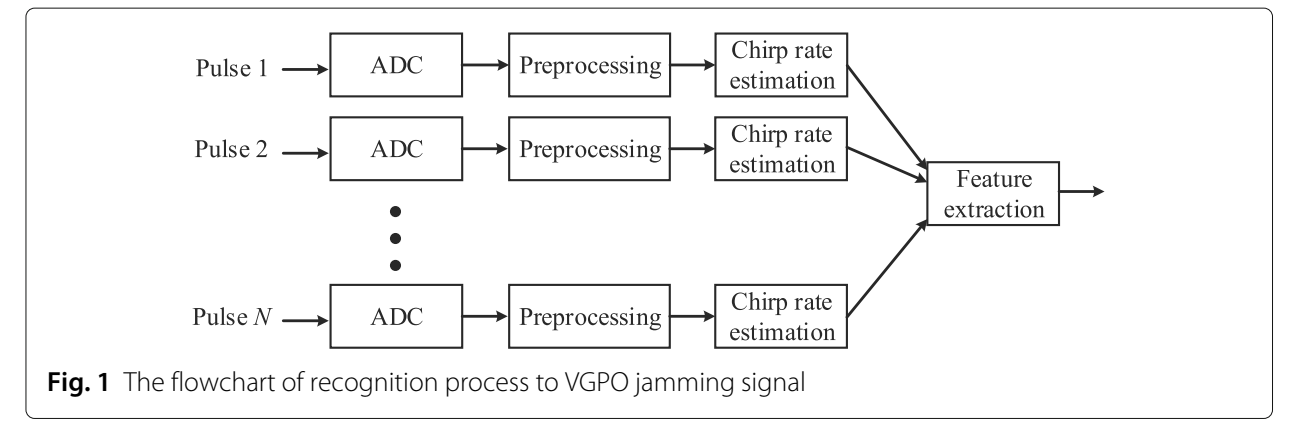

\subsection{Scaling property}

Firstly, we demonstrate the effectiveness of the scaling property of the modified DCFT algorithm. With the acceleration $V_{f} 50 \mathrm{kHz} / \mathrm{s}$ for instance, we consider the scenario that $f_{d j}$ linearly changes with $t$ as shown in (3).

The carrier frequency is $10 \mathrm{GHz}$. The bandwidth and time width of the signal is $5 \mathrm{MHz}$ and $100 \mu \mathrm{s}$, respectively, and the pulse repetition period is $500 \mu \mathrm{s}$. The velocity of the target is fixed as $200 \mathrm{~m} / \mathrm{s}$. The SNR and JNR are $5 \mathrm{~dB}$ and $10 \mathrm{~dB}$, respectively, and the sampling frequency is $10 \mathrm{MHz}$.

Both of the DCFT results of jamming signal and target echo and their corresponding profiles at the frequency where the $\left|X_{m c}^{\prime}\right|^{2}$ maximizes are shown in Fig. 2. In the first row, the scaling factor $C$ is 1 (without scaling). In the second and the third rows, $C$ is $4 e 3$ and $2 e 7$, respectively. In the profiles, both of the modules of the DCFT results of jamming and target are normalized to 1 to compare the estimated chirp rate more visually, and the peaks of DCFT results of jamming and target are respectively signed by a red circle and a blue star.

By comparing the results shown in various rows in Fig. 2, it is easy to find that the DCFT result magnifies in the chirp rate domain as the scaling factor $C$ increases. The estimated chirp rates of target and jamming are distinguishable in Fig. 2i, but not in Fig. 2c and f. Although the estimated chirp rate is quite different from the parameter we set, it is not surprising to us, due to the existence of noise and the quantitative effect.

\subsection{Quantitative effect}

In the second simulation, 500 pulses of jamming signal and target echo are respectively considered to verify the effectiveness of the quantitative effect. The radar signal's parameter is the same as above. Both of the linear and non-linear (quadratic) scenarios in (3) and (26) are considered. The $V_{f}$ is $20 \mathrm{kHz} / \mathrm{s}$, which is equivalent with the acceleration $200 \mathrm{~m} / \mathrm{s}^{2}$ in [28]. The $A_{f}$ is set to $100 \mathrm{kHz} / \mathrm{s}^{2}$ which is relatively large to obtain an intuitional result. The scaling factor of $C$ is $2 \mathrm{e} 5$. The JNR and SNR are $15 \mathrm{~dB}$ and $10 \mathrm{~dB}$, respectively. The estimated values of frequency and chirp rate of the target and linear VGPO in various pulses are shown in Fig. 3a, and the ones of the target and quadratic VGPO are shown in Fig. 3b. For comparison, we also plot the real shifted Doppler frequency of the jamming signal in each pulse.

The estimated frequency of the jamming signal stepwise increases in both linear and quadratic scenarios. In Fig. 3a, the estimated chirp rate of the jamming signal increases linearly from 0 to $0.08 \mathrm{~s}$ and increases quadratically in Fig. 3b, which is coincident with our theoretical analysis. It should be noticed that there always appears a flat area where 


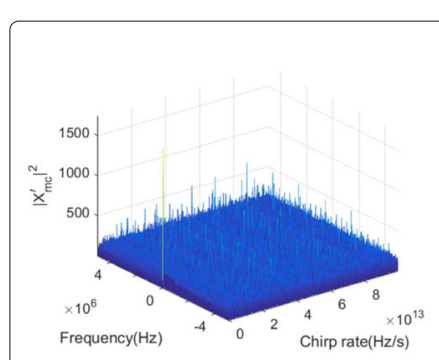

(a)

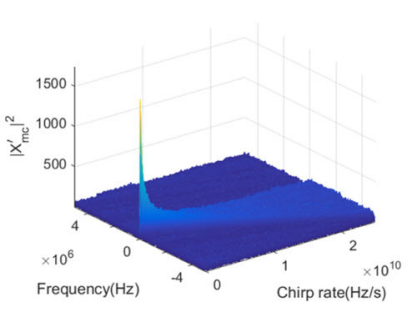

(d)

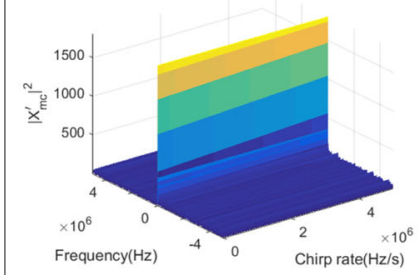

(g)

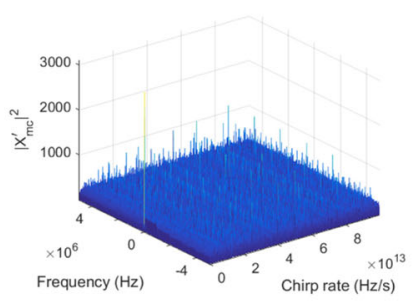

(b)

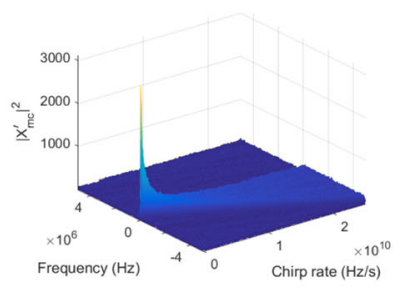

(e)

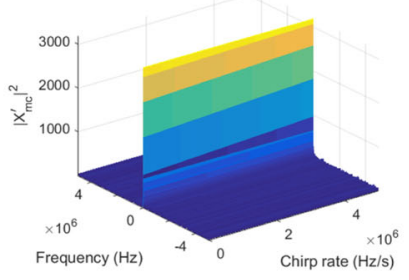

(h)

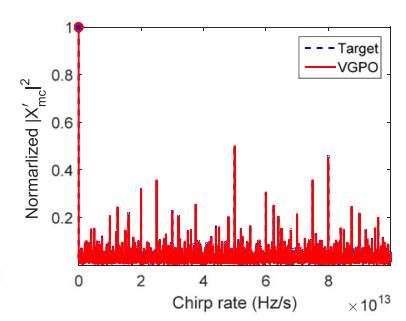

(c)

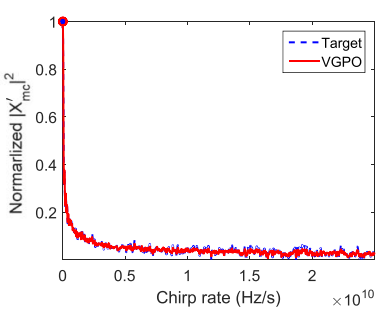

(f)

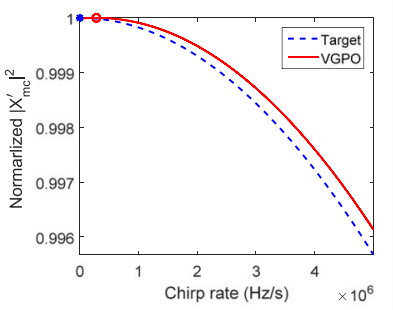

(i)

Fig. 2 The DCFT results of the target echo and VGPO jamming signal with various scaling factor C. a Jamming (without scaling). b Target (without scaling). c Profiles of $\mathbf{a}$ and $\mathbf{b}$ after normalization. $\mathbf{d}$ Jamming $(C=4 \mathrm{e} 3)$. e Target $(C=4 \mathrm{e} 3)$. $\mathbf{f}$ Profiles of $\mathbf{a}$ and $\mathbf{b}$ after normalization. $\mathbf{g}$ Jamming $(C=2 \mathrm{e} 7)$. $\mathbf{h}$ Target $(C=2 \mathrm{e} 7)$. $\mathbf{i}$ Profiles of $\mathbf{g}$ and $\mathbf{h}$ after normalization

the estimated chirp rate is 0 (e.g., from 0.062 to $0.074 \mathrm{~s}$ in Fig. 3b). This is because the estimated frequency increases at this point, that is to say, $\tilde{f}_{d j}$ in (18) is quantized into the next frequency bin. Correspondingly, the remaining Doppler frequency $\Delta f_{m}$ has to subtract $f_{s} / N$, which causes the estimated chirp rate $l$ to become negative. However, the value range of $l$ is from 0 to $N-1$. As a result, the estimated chirp rate $l$ becomes 0 which is the closest one to the theoretical value.

\subsection{Influence of different parameters to DCFT}

We then explore the influence of different signal parameters, pulse numbers, and SNR (or JNR) on identifying the VGPO jamming by utilizing the proposed feature.

We assume the bandwidth of the signal is fixed as $5 \mathrm{MHz}$, and the sampling frequency is $10 \mathrm{MHz}$, while the pulse duration changes from 20 to $100 \mu$ s with duty cycle $20 \%$. The carrier frequency is $15 \mathrm{GHz}$, and the scaling factor $C$ is $2 \mathrm{e} 5$.

To inquire about the question of how long it would take for the proposed method to identify the jamming signal successfully, we jointly consider the influence of the signal's time width and pulse numbers. The linear pull-off scenario is considered. $V_{f}$ is still set to $20 \mathrm{kHz} / \mathrm{s}$. The MSVR of the target echo and the VGPO jamming under various signal parameters and pulse numbers are shown in Fig. $4 \mathrm{a}$ and b, respectively. 

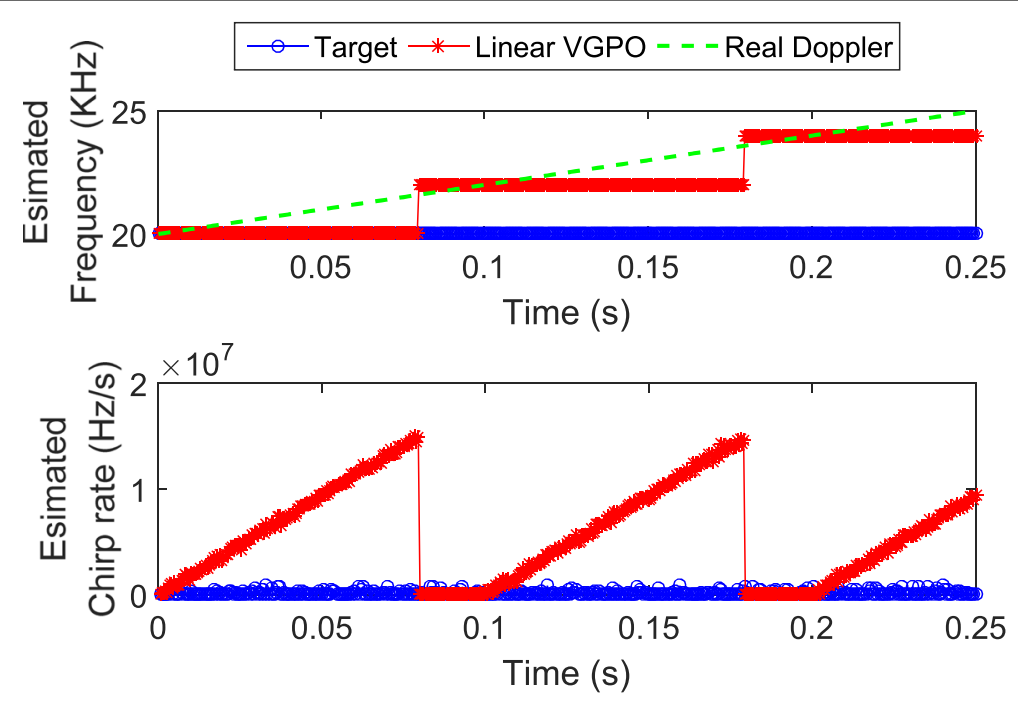

(a)
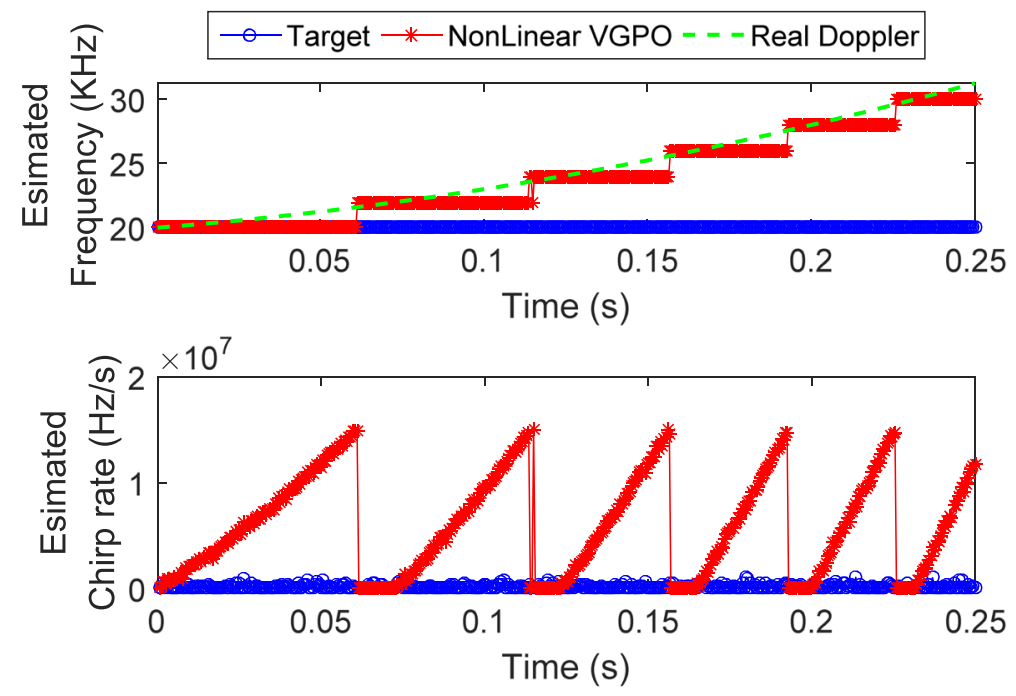

b)

Fig. 3 The estimated frequencies and chirp rates of target echo and jamming signal versus time. $\mathbf{a}$ Linear scenario. b Non-linear scenario

The MSVR of the target echo is steady around 0.45 after 100 pulses, even under different signal time width from Fig. 4a. On the contrary, the MSVR of the VGPO jamming is various under different time width and different pulse numbers in Fig. 4b, and its maximum is about 2.96. By comparing Fig. 4a and b, we can find that the MSVR of jamming signal is always bigger than that of the target echo.

Overall, the MSVR of VGPO jamming increases as the number of pulses increases until the estimated frequency changes. The wider the time width, the faster the value of MSVR increases. It should be noticed that the ladder-shaped portion is caused due to a change in the estimated frequency.

We plot the profiles in Fig. 4b when time width are $50 \mu \mathrm{s}, 80 \mu \mathrm{s}$, and $100 \mu \mathrm{s}$, respectively, and show them in Fig. 5. We also draw the lines corresponding to the number of pulses 


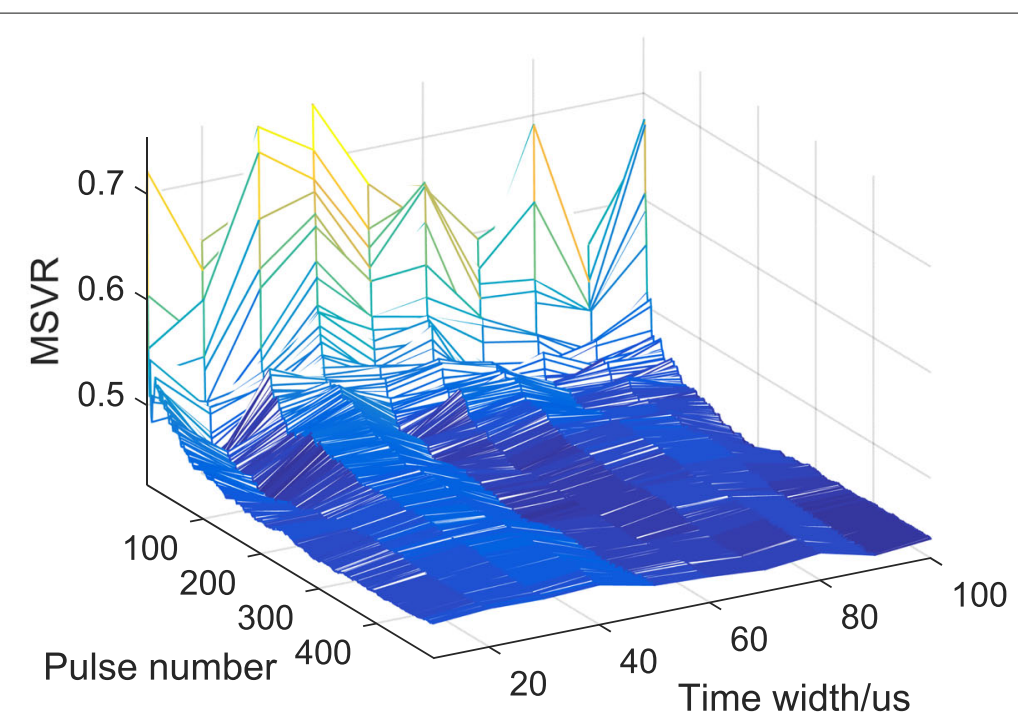

(a)

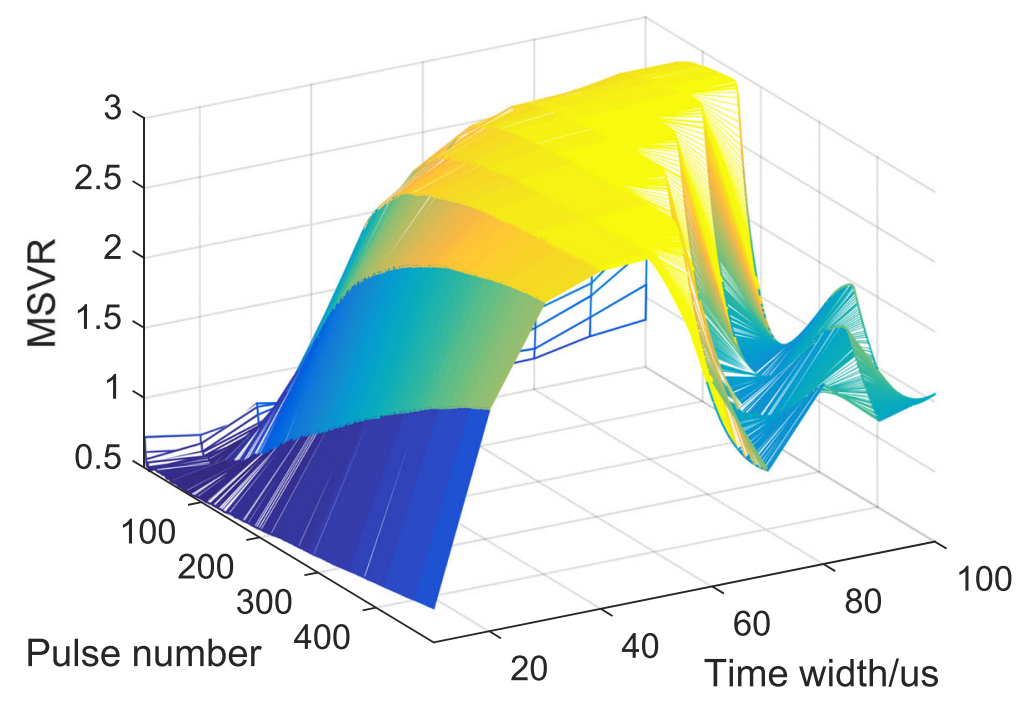

(b)

Fig. 4 The MSVR of target echo and VGPO jamming under different time width and pulse numbers

100,125 , and 200. All intersections formed by the same color represent the same total time of interference implementation $0.05 \mathrm{~s}$, and the MSVR of these points are 2.89, 2.89, and 2.83, respectively. As a result, if we use the number of pulses with the same timestamp (the product of the pulse numbers and PRI) to calculate the characteristic parameter, the MSVR under shorter time width scenario will be smaller as long as the estimated frequency does not change.

We then study the influence of SNR on the performance of the proposed feature. We consider the scenario when the pulse duration is $20 \mu$ s according to the result in Fig. 4b, the total time of interference implementation is $0.08 \mathrm{~s}$ ( 800 pulses).

Besides a target in uniform speed, an accelerated target with an acceleration $2.7 \mathrm{~m} / \mathrm{s}^{2}$, a linear and non-linear pull-off VGPO jamming are also considered. The $V_{f}$ of the linear 


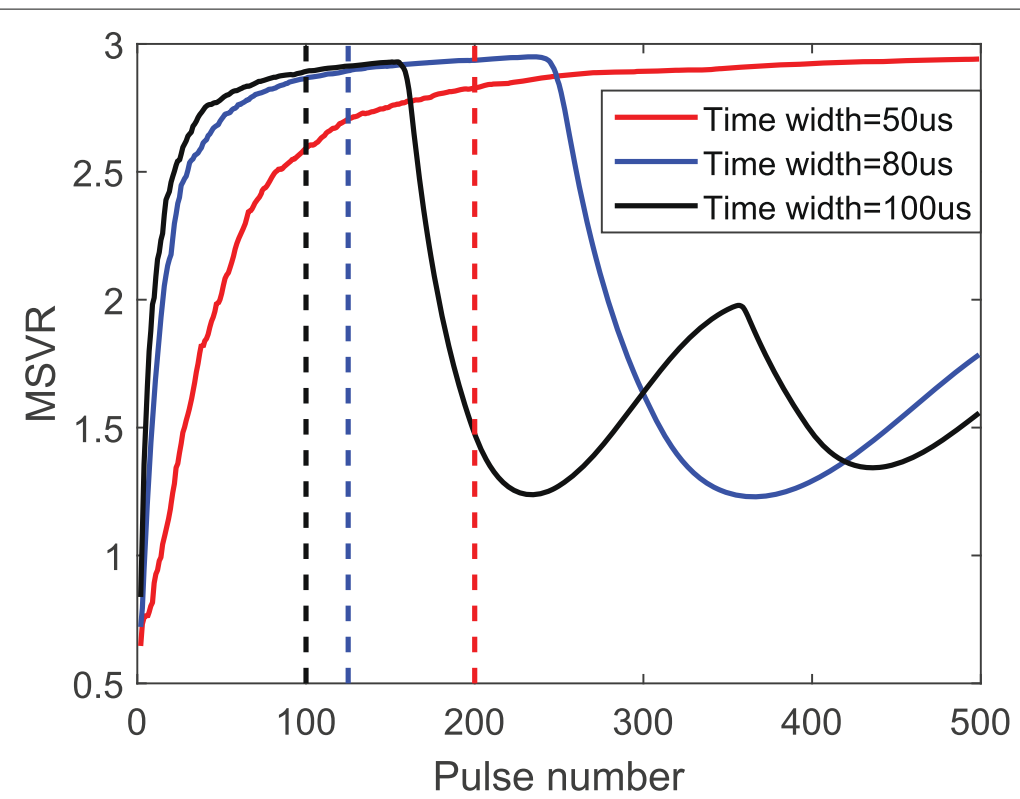

Fig. 5 Profiles of Fig. 4b

pull-off jamming is $2 \mathrm{kHz} / \mathrm{s}$. A non-linear pull-off case is a simple form of the maneuvering scenario, and its $A_{f}$ is $20 \mathrm{kHz} / \mathrm{s}^{2}$. The JSR is fixed as $3 \mathrm{~dB}$, and the SNR changes from 0 to $15 \mathrm{~dB}$. The relationships between MSVR and SNR are shown in Fig. 6.

It can be seen that two kinds of VGPO jamming almost have the same MSVR. It means that the quadratic term of the frequency pull-off function in (26) has less effect on MSVR in a very short time $(0.1 \mathrm{~s})$ if the coefficient of the quadratic term is not too large (e.g., $\left.20 \mathrm{kHz} / \mathrm{s}^{2}\right)$.

Different from the results of the linear and non-linear VGPO jamming, the MSVRs of the target are almost steady which are similar to the result in Fig. 4a. It means

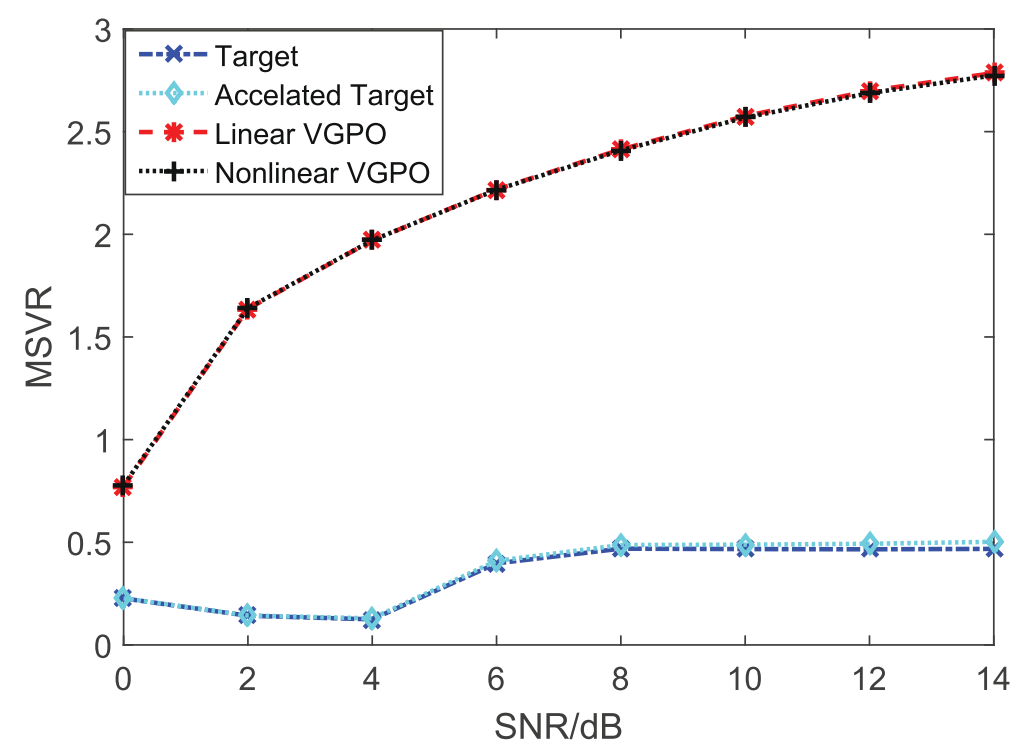

Fig. 6 The MSVR of target echoes and VGPO jamming versus SNR 
that the MSVR of the target signal is unchanged even with various parameters. Moreover, the MSVR of the target and the accelerated target are almost the same. Combined with the MSVR of the two jamming signals, we can conclude that a tiny acceleration or small maneuvering on target hardly changes the value of MSVR.

\subsection{Discrimination performance}

Before we conduct the discrimination performance of the proposed method, we consider the influence of the speed of frequency offset, i.e., the value of $V_{f}$, on MSVR. The sampling frequency is $20 \mathrm{MHz}$, and the SNR and JSR are $10 \mathrm{~dB}$ and $5 \mathrm{~dB}$, respectively. The other parameters are the same as the previous subsection. The values of $V_{f}$ are separately assumed as $20 \mathrm{kHz} / \mathrm{s}$ and $50 \mathrm{kHz} / \mathrm{s}$. The MSVR of target echo and jamming signal versus pulse numbers with various $V_{f}$ are shown in Fig. 7 where VGPO1 and VGPO2 separately denote the linear VGPO jamming with $V_{f}$ of $20 \mathrm{kHz} / \mathrm{s}$ and $50 \mathrm{kHz} / \mathrm{s}$.

The MSVR of the jamming signal with $V_{f}$ of $50 \mathrm{kHz} / \mathrm{s}$ is larger than that of the jamming with $V_{f}$ of $20 \mathrm{kHz} / \mathrm{s}$. Furthermore, the more pulses involved in the calculation, the greater the MSVR of the jamming signal, which is consistent with the result in Fig. 4b.

To compare the performance with the results in [18] and [19], 800 and 850 pulses are separately considered, with the same parameters in Fig. 6. The value of $V_{f}$ is set as $20 \mathrm{kHz} / \mathrm{s}$, and 300 Monte Carlo trials are considered. As the value of MSVR of the target signal is stable under different radar parameters, the threshold can be set as the average between the minimum MSVR of the jamming signal, which is usually the corresponding value when the SNR is $0 \mathrm{~dB}$, and the stable value of MSVR of the target. In this simulation, the threshold is set as 0.65 as the MSVR of jamming signal is around 0.8 when the SNR is $0 \mathrm{~dB}$ in Fig. 6. Figure 8 shows the identification probability of jamming signal versus SNR based on the proposed method.

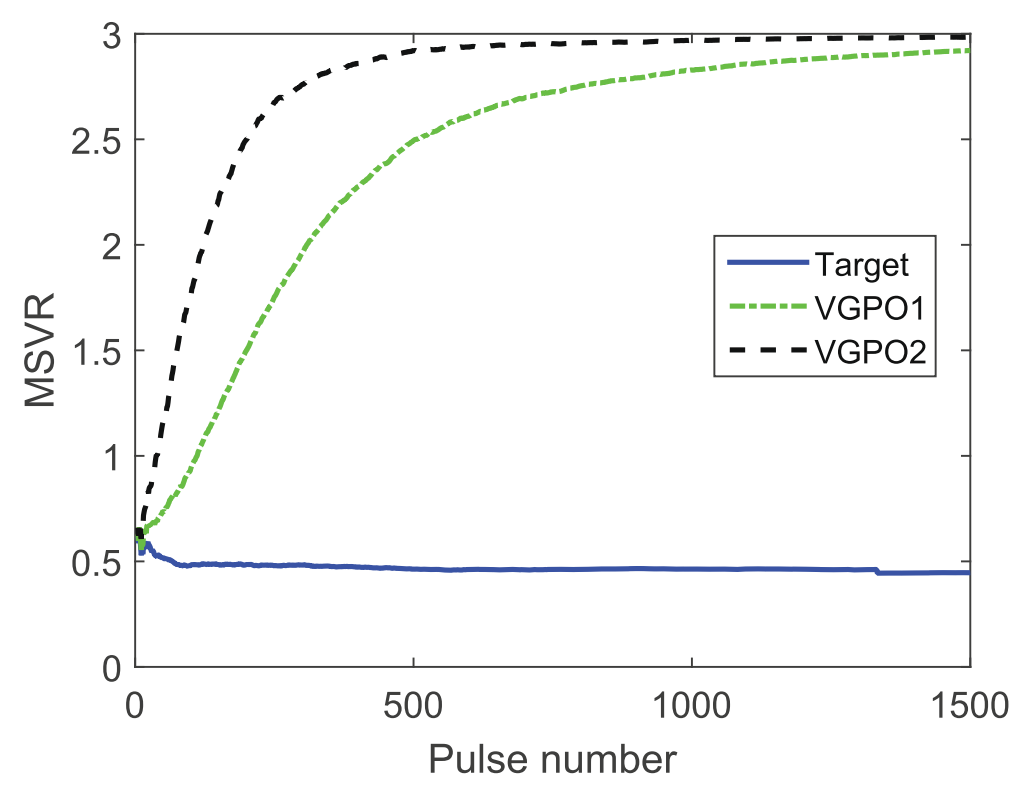

Fig. 7 The MSVR of the target echo and two kinds of jamming signals versus pulse number 


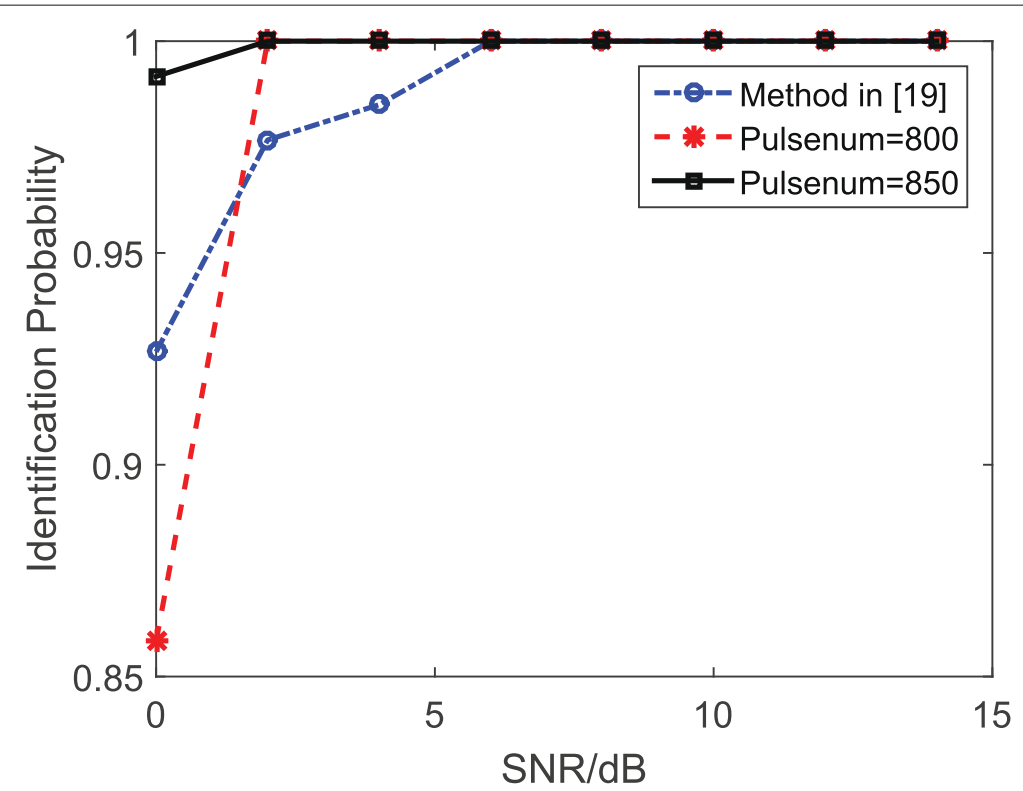

Fig. 8 The identification probabilities of the proposed method under different pulse numbers and the method in [19]

From Fig. 8, when the SNR is $0 \mathrm{~dB}$, the identification probability of VGPO jamming could reach 0.975 with 850 pulses and 0.81 with 800 pulses. When the SNR is $2 \mathrm{~dB}$, the jamming signal can always be correctly recognized.

From the aspect of the total data needed to correctly identify the jamming signal, our method only uses the signal data within $0.085 \mathrm{~s}$ and is less than the method in [18] which needs the signal data within $0.096 \mathrm{~s}$. From the aspect of the identification probability, our method can correctly recognize the jamming signal when the SNR is $2 \mathrm{~dB}$, which is smaller than that in [18] (the blue dotted line in Fig. 8). It should be noted that the value of $V_{f}$ is $50 \mathrm{kHz} / \mathrm{s}$ in [18], which also means our method would be better if the same value of $V_{f}$ is used based on the result in Fig. 7.

\subsection{Discussion}

Although this method can recognize the VGPO jamming with less data and a higher recognition rate compared to the open literature, it is not suitable for the jamming of range gate pull-off (RGPO) with uniform acceleration. This is because its chirp rate after de-chirping is pretty small (about 0.2 for the false target with the same motion state with the same simulation parameters in this paper). However, as a more general form of VGPO, the range-velocity gate pull-off (R-VGPO) jamming can also be identified by this method. Even in mixed-signal including target echo and jamming, this method is still effective.

\section{Conclusions}

In this paper, the discrimination issue between the target echo and the VGPO jamming is studied. To this purpose, we resort the discrete chirp-Fourier transform to jointly estimate the chirp rate and frequency of a signal, and the scaling characteristic of the DCFT algorithm is studied and explored to increase the estimated accuracy in chirp rate 
domain. Based on the actual joint estimation results, the quantitative effect of the VGPO signal is further analyzed; it guarantees that the relationship between the pull-off acceleration and the time can be judged from the relationship between the estimated chirp rate and the pulse numbers. To distinguish VGPO jamming and target echo, the MSVR is defined. Simulation results indicate that for a timestamp when the estimated frequency is unchanged, the MSVR of VGPO jamming increases with the pulse numbers and time width (with the same duty cycle) increases and is always larger than that of a target which is stable. Compared to other methods, the proposed method can recognize the jamming signal with less data and have a higher recognition; it means a great potential in practical applications.

\section{Abbreviations}

VGPO: Velocity gate pull-off; DCFT: Discrete chirp-Fourier transform; MSVR: The mean square to variance ratio; JNR: Jamming-to-noise ratio; ECM: Electronic countermeasure; DRFM: Digital radio frequency memory; ECCM: Electronic counter-countermeasures; RGPO: Range gate pull-off; PRI: Pulse repetition interval; DFT: Discrete Fourier transform; SNR: Signal-to-noise ratio; JSR: Jamming-to-signal ratio; LFM: Linear frequency modulation

\section{Acknowledgements}

Thanks to the teachers and the students of the research group for valuable discussion and all the people who had put forward valuable suggestions.

\section{Authors' contributions}

Chuanzhang Wu found the idea and wrote this article, and Baixiao Chen put forward some constructive suggestions for revision. The authors read and approved the final manuscript.

\section{Funding}

Not applicable.

Availability of data and materials

Not applicable.

Ethics approval and consent to participate

Not applicable.

\section{Consent for publication}

Not applicable.

\section{Competing interests}

The authors declare that they have no competing interests.

Received: 1 April 2020 Accepted: 20 July 2020

Published online: 17 August 2020

\section{References}

1. D. C. Schleher, Electronic Warfare in the Information Age, 1st edn. (Artech House, Inc., USA, 1999)

2. R. A. Poisel, Information Warfare and Electronic Warfare Systems. (Artech House, Inc., Norwood, 2013)

3. L. N. Jing, Z. Y. Ting, A survey of radar ECM and ECCM. IEEE Trans. Aerosp. Electron. Syst. 31(3), 1110-1120 (1995). https://doi.org/10.1109/7.395232

4. S. J. Roome, Digital radio frequency memory. Electron. Commun. Eng. J. 2(4), 147-153 (1990). https://doi.org/10. 1049/ecej:19900035

5. M. Greco, F. Gini, A. Farina, V. Ravenni, Effect of phase and range gate pull-off delay quantisation on jammer signal. IEE Proc. Radar Sonar Navig. 153(5), 454-459 (2006)

6. M. Greco, F. Gini, A. Farina, Radar detection and classification of jamming signals belonging to a cone class. IEEE Trans. Signal Proc. 56(5), 1984-1993 (2008)

7. F. Bandiera, D. Orlando, G. Ricci, Advanced Radar Detection Schemes Under Mismatched Signal Models. (USA, Morgan \& Claypool, 2009)

8. F. Bandiera, A. Farina, D. Orlando, G. Ricci, Detection algorithms to discriminate between radar targets and ECM signals. IEEE Trans. Signal Process. 58(12), 5984-5993 (2010)

9. V. Carotenuto, A. De Maio, D. Orlando, L. Pallotta, Adaptive radar detection using two sets of training data. IEEE Trans. Signal Process. 66(7), 1791-1801 (2018)

10. L. Yan, P. Addabbo, C. Hao, D. Orlando, A. Farina, New ECCM techniques against noiselike and/or coherent interferers. IEEE Trans. Aerosp. Electron. Syst. 56(2), 1172-1188 (2020) 
11. J. Xu, G. Liao, S. Zhu, H. C. So, Deceptive jamming suppression with frequency diverse MIMO radar. Signal Process. 113(C), 9-17 (2015). https://doi.org/10.1016/j.sigpro.2015.01.014

12. S. Zhao, L. Zhang, Y. Zhou, N. Liu, Signal fusion-based algorithms to discriminate between radar targets and deception jamming in distributed multiple-radar architectures. IEEE Sensors J. 15(11), 6697-6706 (2015). https://doi. org/10.1109/JSEN.2015.2440769

13. S. Zhao, Y. Zhou, L. Zhang, Y. Guo, S. Tang, Discrimination between radar targets and deception jamming in distributed multiple-radar architectures. let Radar Sonar Navig. 11(7), 1124-1131 (2017). https://doi.org/10.1049/ietrsn.2016.0540

14. Q. Sun, T. Shu, K. Yu, W. Yu, Efficient deceptive jamming method of static and moving targets against SAR. IEEE Sensors J. 18(9), 3610-3618 (2018)

15. W. Li, W. Liu, L. Guo, X. Yang, in IET International Radar Conference 2015, Joint slow/fast-time pulse diversity method for countering range gate pull off jamming, (2015), pp. 1-4. https://doi.org/10.1049/cp.2015.1483

16. J. Zhang, D. Zhu, G. Zhang, New antivelocity deception jamming technique using pulses with adaptive initial phases. Aerosp. Electron. Syst. IEEE Trans. 49(2), 1290-1300 (2013). https://doi.org/10.1109/TAES.2013.6494414

17. W. Xiong, X. Wang, G. Zhang, in 2016 IEEE Radar Conference (RadarConf), Cognitive waveform design for anti-velocity deception jamming with adaptive initial phases, (2016), pp. 1-5. https://doi.org/10.1109/radar.2016.7485306

18. L. Wu, X. Fu, S. Liu, S. Peng, M. Xie, in 2016 CIE International Conference on Radar (RADAR), The anti-jamming method based on front-back-edge tracking of VGPO jamming, (2016), pp. 1-4. https://doi.org/10.1109/radar.2016.8059170

19. Y. Xingyu, R. Huailin, F. Haoran, in 2017 th IEEE International Conference on Electronics Information and Emergency Communication (ICEIEC), A recognition algorithm of deception jamming based on image of time-frequency distribution, (2017), pp. 275-278. https://doi.org/10.1109/iceiec.2017.8076561

20. T. Abatzoglou, in ICASSP '86. IEEE International Conference on Acoustics, Speech, and Signal Processing, vol. 11, Fast maximum likelihood joint estimation of frequency and frequency rate, (1986), pp. 1409-1412

21. S. Qian, D. Chen, Q. Yin, in Proceedings of the 1998 IEEE International Conference on Acoustics, Speech and Signal Processing, ICASSP'98 (Cat. No.98CH36181), vol. 3, Adaptive chirplet based signal approximation, (1998), pp. 1781-17843. https://doi.org/10.1109/icassp.1998.681805

22. P. Wang, H. Li, H. Djurovic, Performance of instantaneous frequency rate estimation using high-order phase function. IEEE Trans. Signal Process. 58(4), 2415-2421 (2010). https://doi.org/10.1109/TSP.2009.2034939

23. X. G. Xia, Discrete chirp-fourier transform and its application to chirp rate estimation. IEEE Trans. Signal Process. 48(11), 3122-3133 (2000). https://doi.org/10.1109/78.875469

24. X. Guo, H. B. Sun, S. L. Wang, G. S. Liu, Comments on discrete chirp-fourier transform and its application to chirp rate estimation [with reply]. Signal Process. IEEE Trans. 50(12), 3115-3116 (2002). https://doi.org/10.1109/TSP.2002. 805492

25. Y. An, B. Tian, Y. Sun, in 2008 9th International Conference on Signal Processing, An interference suppression algorithm based on a novel MDCFT method for DSSS system, (2008), pp. 1858-1862. https://doi.org/10.1109/icosp.2008. 4697503

26. S. D. Berger, Digital radio frequency memory linear range gate stealer spectrum. IEEE Trans. Aerosp. Electron. Syst. 39, 1791-1801 (2003)

27. L. Bo, S. Yao, Z. Chang-you, in 2011 International Conference on Electronics, Communications and Control (ICECC), Study of multistatic radar against velocity-deception jamming, (2011), pp. 1044-1047. https://doi.org/10.1109/icecc.2011. 6066436

28. J. D. Townsend, M. A. Saville, S. M. Hongy, R. K. Martin, in 2008 IEEE Radar Conference, Simulator for velocity gate pull-off electronic countermeasure techniques, (2008), pp. 1-6. https://doi.org/10.1109/radar.2008.4720888

29. P. Fan, X.-G. Xia, in WCC 2000 - ICSP 2000. 2000 5th International Conference on Signal Processing Proceedings. 16th World Computer Congress 2000, vol. 1, A modified discrete chirp-Fourier transform scheme, (2000), pp. 57-601. https://doi. org/10.1109/icosp.2000.894443

\section{Publisher's Note}

Springer Nature remains neutral with regard to jurisdictional claims in published maps and institutional affiliations.

\section{Submit your manuscript to a SpringerOpen ${ }^{\circ}$ journal and benefit from:}

- Convenient online submission

- Rigorous peer review

Open access: articles freely available online

- High visibility within the field

- Retaining the copyright to your article

Submit your next manuscript at $\gg$ springeropen.com 